\title{
ON THE PADÉ APPROXIMANTS ASSOCIATED WITH THE CONTINUED FRACTION AND SERIES OF STIELTJES*
}

\author{
HUBERT S. WALL
}

\section{INTRODUCTION}

The well known series of Stieltjes is a power series with real coefficients:

$$
\frac{c_{0}}{z}-\frac{c_{1}}{z^{2}}+\frac{c_{2}}{z^{3}}-\cdots
$$

such that the persymmetric determinants

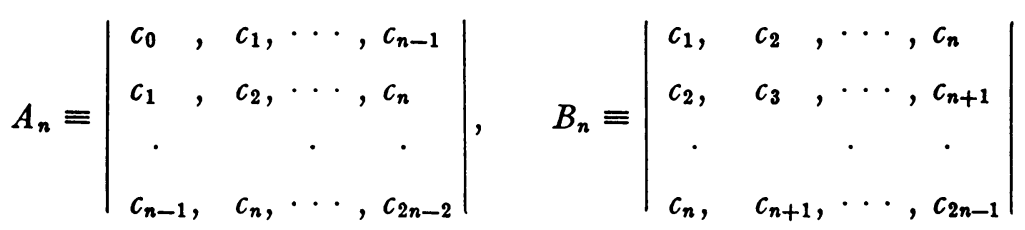

$\left(n=1,2,3, \cdots, A_{0} \equiv 1, B_{0} \equiv 1\right)$ are all positive. Stieltjes $\dagger$ connected the series (1) with a continued fraction

$$
\frac{1}{a_{1} z}+\frac{1}{a_{2}}+\frac{1}{a_{3} z}+\cdots
$$

in which all the $a_{i}$ are real and positive, and are uniquely determined by the $c_{i}$ in accordance with the relations

$$
a_{2 n}=A_{n}^{2} /\left(B_{n} B_{n-1}\right), \quad a_{2 n+1}=B_{n}^{2} /\left(A_{n} A_{n+1}\right) .
$$

Conversely, the positive real numbers $a_{i}(i=1,2,3, \ldots)$ uniquely determine coefficients $c_{i}$ of a Stieltjes power series. Thus to every Stieltjes continued fraction (2) with positive $a_{i}$ there corresponds a Stieltjes series (1) and vice versa.

In the work of Stieltjes the convergents of (2) separate into two sets, the odd and the even convergents. When the series $\ddagger \sum a_{i}$ is divergent, these

* Presented to the Society, April 16, 1927; received by the editors June 11, 1928. This paper is essentially a thesis prepared at the suggestion of Professor E. B. Van Vleck at the University of Wisconsin.

$\dagger$ Recherches sur les fractions continues, Annales de Toulouse, vol. 8, J, pp. 1-122, and vol. 9, A, pp. 1-47, 1894-95. Published also in vol. 32 of the Mémoires présentés a l'Académie des Sciences de l'Institut National de France.

$\ddagger$ Here and henceforth we write $\sum$ in place of $\sum_{i=1}^{\infty}$. 
two sets of alternate convergents have one and the same limit which is a function analytic throughout the complex plane, excepting the whole or a part of the negative real axis. When, on the other hand, the series $\sum a_{i}$ converges, these two sets have distinct limits $p_{1} / q_{1}$ and $p / q$ respectively, which are meromorphic functions having only simple poles all of which are real and non-positive. The numerators and denominators $p, p_{1}, q, q_{1}$ are analytic functions between which there is the relation

$$
p_{1} q-p q_{1}=+1 \text {. }
$$

In the case of a convergent Stieltjes series (1) the former alternative is always realized, but for a divergent series either alternative may be realized. Thus for some divergent series the continued fraction of Stieltjes picks out two functional equivalents, while for others only a single equivalent is yielded.

In this article it will be convenient to replace $1 / z$ by $z$ in (1) and (2) and then drop the unessential factor $z$. We then have the Stieltjes series

$$
c_{0}-c_{1} z+c_{2} z^{2}-c_{3} z^{3}+\cdots
$$

with the same inequalities:

$$
A_{n}>0, \quad B_{n}>0,
$$

while the continued fraction becomes

$$
\frac{1}{a_{1}}+\frac{z}{a_{2}}+\frac{z}{a_{3}}+\frac{z}{a_{4}}+\cdots
$$

When $\sum a_{i}$ is convergent, the two sets of alternate convergents have limits which are distinct and meromorphic over the entire plane except at the origin, while if $\sum a_{i}$ is divergent both sets have the same limit which is a function analytic over the entire plane except the whole or a part of the negative real axis.

With any power series

$$
k_{0}+k_{1} z+k_{2} z^{2}+\cdots
$$$$
\left(k_{0} \neq 0\right) \text {, }
$$

Padé connects a table of approximants*

$$
\begin{array}{l|l|l}
\cline { 2 - 3 } m=0 & n=0 & n=1 \\
\hline \frac{N_{0,0}}{D_{0,0}}=k_{0}, & \frac{N_{0,1}}{D_{0,1}}=k_{0}+k_{1} z, & \ldots \\
\hline \frac{N_{1,0}}{D_{1,0}}, & \frac{N_{1,1}}{D_{1,1}}, & \ldots \\
\hline \cdots & \cdots & \cdots
\end{array}
$$

* Pade, Thesis, published in the Annales de l'Ecole Normale Supérieure, (3). vol 9. supplement, pp. 1-93, 1892. 
where $N_{m, n}(z) / D_{m, n}(z)$ is the rational fraction, taken in its lowest terms, in which the degrees of numerator and denominator do not exceed $n$ and $m$ respectively, and whose expansion into a power series, $P(z)$, agrees with (8) for a greater number of terms than any other such rational fraction.

If a particular approximant does not appear more than once in the table, that approximant is said to be normal, and when every approximant is normal, the table is called normal. The power series $P(z)$ for a normal approximant, in which necessarily the degrees of numerator and denominator are exactly $n$ and $m$ respectively, agrees with the given series term by term up to and including the term of degree $m+n$ and no further.

Professor Van Vleck* showed that the series of Stieltjes (5) has a normal Padé table. The odd convergents of the continued fraction (7) fill the principal diagonal of the table, while the even convergents fill the parallel diagonal file immediately below it. Consequently these two diagonal files of approximants will either converge to a common limit analytic throughout the entire plane except over the whole or a part of the negative real axis, or to two distinct functions, meromorphic everywhere except at the origin, according as $\sum a_{i}$ diverges or converges.

The first main question which $I$ have considered in this article is the following:

1. What holds regarding the convergence of the other diagonal files parallel to the principal diagonal in the Pade table for the series of Stieltjes?

I find that the sequence of convergents in any file parallel to the principal diagonal converges to a limit. Three cases arise.

Case I. If the series $\sum a_{i}$ is convergent, no two files to the right of and parallel to the principal diagonal have the same limit, and no two parallel files below the principal diagonal have the same limit. In this case all the limit functions are meromorphic over the entire plane except at the origin. The poles of the limits of the files to the right of the principal diagonal are all simple and lie upon the negative half of the real axis.

When $\sum a_{i}$ diverges there are two possibilities which exist, namely:

Case II. The file of approximants in the principal diagonal and the successive parallel files to the right of it up to the $n$th file inclusive converge to one and the same limit, while subsequent to the $n$th they converge to distinct limits. Likewise the successive parallel files below the principal diagonal, down to an $m$ th file inclusive, may converge to the same limit, while subsequent to the $m$ th file they converge to distinct limits.

* On an extension of the 1894 memoir of Stieltjes, these Transactions, vol. 4 (1903), pp. 297-332. 
$A l l$ the limit functions in this case are meromorphic over the entire plane except at the origin.

Case III. Every diagonal file parallel to the principal diagonal converges to one and the same function, which is analy tic over the entire plane excepting the whole or a part of the negative half of the real axis.

In Case I, the series (5) diverges, while in Cases II and III it may either diverge or converge. Whereas Stieltjes obtained two distinct functional equivalents of the divergent series (5) in Case I, we now have an infinite set; and whereas Stieltjes obtained a single functional equivalent when $\sum a_{i}$ diverges, I obtain an infinite number in Case II, and but one in Case III.

Generalizing (1), I shall call any series

$$
\pm\left(c_{0} z^{n}-c_{1} z^{n+1}+c_{2} z^{n+2}-\cdots\right),
$$

in which $n$ is a positive or negative integer or zero, a Stieltjes series if the coefficients $c_{i}$ satisfy the Stieltjes inequalities (6).

If the first $n$ terms of (5) be removed, the remainder is still a Stieltjes series. Instead of removing terms I have concerned myself with the following question:

2. Can the series (5) be so continued to the left by successive addition of new terms that the extended series shall be Stieltjes series?

I find that the necessary and sufficient condition for a first extension is that the series $\sum a_{2 i}$, with even subscripts, shall converge.

Two cases arise:

Case A. If the series $\sum a_{i}$ converges, the Stieltjes series (5) may be extended to the left by the addition of an arbitrary number of new terms, forming thereby a series whose coefficients satisfy the Stieltjes inequalities (6). The coefficients of the prefixed terms are not unique.

Case B. If the series $\sum a_{i}$ diverges and $\sum a_{2 i}$ converges, the series (5) may be extended by the addition of a term, $-c_{-1} z^{-1}$, in which $c_{-1}$ may be taken equal to or greater than $\sum a_{2 i}$. If $c_{-1}>\sum a_{2 i}$, further extension is never possible; but if $c_{-1}=\sum a_{2 i}$, the like series $\sum a_{2 i}^{\prime}$ in the new Stieltjes continued fraction belonging to the extended series may or may not converge. If it does converge, a second extension is possible. The coefficient of the new term, $c_{-2} z^{-2}$, prefixed may be taken equal to or greater than $\sum a_{2 i}^{\prime}$. Only if $c_{-2}$ $=\sum a_{2 i}^{\prime}$, can a third term be prefixed, and so on.

When $n$ terms can be prefixed, all the coefficients in the added terms are unique with the exception of $c_{-n}$. For an infinite extension all the coefficients are unique. There exist series which can be extended to just $n$ terms and others which can be infinitely extended. 


\section{Chapter I. The Pade table of approximants}

To each pair of non-negative integers $(m, n)$ there corresponds one rational function of the complex variable $z=x+i y: N_{m, n}(z) / D_{m, n}(z)$ taken in its lowest terms, in which the degrees of numerator and denominator do not exceed $n$ and $m$ respectively, and whose expansion into a series in ascending powers of $z$ agrees term by term with a given series

$$
k_{0}+k_{1} z+k_{2} z^{2}+\cdots
$$

to a higher degree than all other such rational functions. In fact, if we let $N_{m, n}(z)=s_{0}+s_{1} z+\cdots+s_{n} z^{n}$ and $D_{m, n}(z)=t_{0}+t_{1} z+\cdots+t_{m} z^{m}$, the conditions that the series

$$
\left(k_{0}+k_{1} z+\cdots\right)\left(t_{0}+t_{1} z+\cdots+t_{m} z^{m}\right)-\left(s_{0}+s_{1} z+\cdots+s_{n} z^{n}\right)
$$

shall begin with the $(m+n)$ th power of $z$ are the following:

$$
\begin{aligned}
k_{i} t_{0}+k_{i-1} t_{1}+\cdots+k_{i-m} t_{m}=s_{i} & \\
(i & \left.=0,1, \cdots, n ; \quad k_{j}=0 \text { if } j<0\right), \\
k_{n+i} t_{0}+k_{n+i-1} t_{1}+\cdots+k_{n+i-m} t_{m}=0 & \\
(i & \left.=1,2, \cdots, m ; \quad k_{j}=0 \text { if } j<0\right) .
\end{aligned}
$$

If we take $t_{0}$ equal to the determinant

$$
\Delta_{m-1, n} \equiv\left|\begin{array}{ccc}
k_{n-m+1} & \cdots & k_{n} \\
\cdot & \cdot & \cdot \\
k_{n} & \cdots & k_{n+m-1}
\end{array}\right|
$$

and assume $\Delta_{m-1, n} \neq 0$, then all the other $t_{i}$ and also the $s_{i}$ are determined uniquely, and $N_{m, n}$ and $D_{m, n}$ may be written in the form of determinants:

$$
\begin{aligned}
& N_{m, n}(z)=\left|\begin{array}{cccc}
k_{n-m+1} \cdots & k_{n} & k_{n-m} z^{n}+k_{n-m-1} z^{n-1}+\cdots+k_{0} z^{m} \\
\cdot & \cdot & \cdot & \cdot \\
k_{n+1} & \cdots & k_{n+m} & k_{n} z^{n}+k_{n-1} z^{n-1}+\cdots+k_{0}
\end{array}\right|, \\
& D_{m, n}(z)=\left|\begin{array}{cccc}
k_{n-m+1} & \cdots & k_{n} & z^{m} \\
\cdot & \cdot & \cdot & \\
k_{n+1} & \cdots & k_{n+m} & 1
\end{array}\right|
\end{aligned}
$$

The table of approximants is normal when and only when all the determinants

$$
\Delta_{m, n} \equiv\left|\begin{array}{ccc}
k_{n-m} & \cdots & k_{n} \\
\cdot & \cdot & \cdot \\
k_{n} & \cdots & k_{n+m}
\end{array}\right| \quad\left(\begin{array}{r}
m, n=0,1,2, \cdots \\
k_{i}=0, \text { for } i<0
\end{array}\right)
$$


are different from zero.* We will henceforth assume the normality of the table.

In a normal table Padé distinguished three types of regular continued fractions. We shall need to use here the stairlike type. This is obtained by starting from a border element of the table of approximants, and passing hence alternately one step to the right and one downward or vice versa. If, for example, we start from the $n$th element of the upper border of the table and proceed hence one step to the right and one down alternately, we get the sequence of approximants

$$
\frac{N_{0, n-1}(z)}{D_{0, n-1}(z)}, \frac{N_{0, n}(z)}{D_{0, n}(z)}, \frac{N_{1, n}(z)}{D_{1, n}(z)}, \frac{N_{1, n+1}(z)}{D_{1, n+1}(z)}, \ldots
$$

whose terms are the successive convergents of the continued fraction

$$
k_{0}+k_{1} z+k_{2} z^{2}+\cdots+k_{n-1} z^{n-1}+z^{n}\left[\frac{1}{h_{1}^{(n)}}+\frac{z}{h_{2}^{(n)}}+\frac{z}{h_{3}^{(n)}}+\cdots\right],
$$

in which

$$
h_{2 i}^{(n)}=-\frac{\Delta_{i-1, i+n-1}^{2}}{\Delta_{i-1, i+n} \Delta_{i-2, i+n-1}}, \quad h_{2 i+1}^{(n)}=\frac{\Delta_{i-1, i+n}^{2}}{\Delta_{i-1, i+n-1} \Delta_{i, i+n}} .
$$

If we start with the corner element of the table and proceed hence one step downward and one to the right alternately, we get the sequence of approximants

$$
\frac{N_{0,0}(z)}{D_{0,0}(z)}, \frac{N_{1,0}(z)}{D_{1,0}(z)}, \frac{N_{1,1}(z)}{D_{1,1}(z)}, \frac{N_{2,1}(z)}{D_{2,1}(z)}, \ldots,
$$

whose terms are the successive convergents of a continued fraction

in which

$$
\frac{1}{a_{1}}+\frac{z}{a_{2}}+\frac{z}{a_{3}}+\frac{z}{a_{4}}+\cdots
$$

$$
a_{2 i}=-\frac{\Delta_{i-1, i-1}^{2}}{\Delta_{i-1, i} \Delta_{i-2, i-1}}, \quad a_{2 i+1}=\frac{\Delta_{i-1, i}^{2}}{\Delta_{i-1, i-1} \Delta_{i, i}} .
$$

It is to be understood that $\Delta_{-1,0}$ is to be taken here and hereafter equal to unity.

If now the continued fraction (16) converges, the two adjacent diagonal files of the table from which (15) is obtained by selecting approximants from each alternately, converge to one and the same limit, namely the limit of (16). On the other hand, if these two diagonal files converge to separate

* For details, see Padé, Thesis, loc. cit; or O. Perron, Die Lehre von den Kettenbrüchen, 1913, Chap. X, pp. 418-465. 
limits, the sequences of alternate convergents of (16) must have separate limits.

It is easily seen that not only does the series (8) uniquely determine the continued fraction (19) in the case of a normal table but conversely (19) determines the series. The study of the convergence of the files of approximants parallel to the principal diagonal of the table can therefore be made to depend upon the sequence of numbers $a_{i}$ in (19). In the next chapter such a study is made for the diagonal files to the right of the principal diagonal of the table in the case of the continued fraction of Stieltjes.

To investigate the convergence of the diagonal files below the principal diagonal which start on the left border of the table, I find it desirable to turn to the Padé table of the reciprocal series

$$
\delta_{0}+\delta_{1} z+\delta_{2} z^{2}+\delta_{3} z^{3}+\cdots,
$$

which is determined by the identity

$$
\left(k_{0}+k_{1} z+\cdots\right)\left(\delta_{0}+\delta_{1} z+\cdots\right)=1 .
$$

The approximant corresponding to $(m, n)$ for this series is the reciprocal of the approximant corresponding to $(n, m)$ for the original series (8); and when, as supposed, the Padé table of (8) is normal, the table of the reciprocal series is also normal, inasmuch as the first $m+n+1$ terms of the latter series are determined by the first $m+n+1$ terms of the former and conversely. Thus the question of the convergence of the diagonal files to the left of the principal diagonal in a normal Pade table may be replaced by the consideration of the convergence of the diagonal files to the right of the principal diagonal in the table of the reciprocal series. Then

Let now $N_{n, m}^{\prime}(z) / D_{n, m}^{\prime}(z), m>n$, be an approximant of the series (20).

$$
\begin{aligned}
& D_{n, m}^{\prime}=w(m, n-m) N_{m, n}, \\
& N_{n, m}^{\prime}=w(m, n-m) D_{m, n},
\end{aligned}
$$

where $w(m, n-m)$ is a numerical factor independent of $z$. By (13), (14) the relation (22) may be written

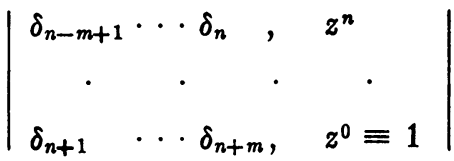

$$
\begin{aligned}
& =w(m, n-m)\left|\begin{array}{cccc}
k_{m-n+1} & \cdots & k_{m}, & k_{m-n} z^{m}+k_{m-n-1} z^{m-1}+\cdots \\
\cdot & \cdot & \cdot & \cdot \\
k_{m+1} & \cdots & k_{m+n}, & k_{m} z^{m}+k_{m-1} z^{m-1}+\cdots
\end{array}\right| \text {, }
\end{aligned}
$$

with a similar equation resulting from (23). 
On setting $z=0$, and replacing $n$ by $n+m$ in (24) the latter becomes

$$
\Delta_{m-1, n+m}^{\prime}=k_{0} w(m, n) \cdot \Delta_{m+n^{-}} \quad m,
$$

where $\Delta_{m, n}^{\prime}$ denotes the determinant $\Delta_{m, n}$ expressed in terms of the $\delta_{i}$ instead of the $k_{i}$.

We will now determine the constant $w(m, n)$.

From the identity (21) we have

which gives

$$
\begin{aligned}
\delta_{0} k_{0} & =1, \\
\delta_{1} k_{0}+\delta_{0} k_{1} & =0, \\
\cdot \quad \cdot & \cdot \\
\delta_{n} k_{0}+\delta_{n-1} k_{1}+\cdots+\delta_{0} k_{n} & =0,
\end{aligned}
$$

$$
\delta_{n}=(-1)^{n(n+1) / 2} \cdot \frac{1}{k_{0}^{n+1}} \cdot \Delta_{n-1,1} .
$$

Also, setting $m=1$ and replacing $n$ by $n-1$ in (25) we have

$$
\delta_{n}=k_{0} w(1, n-1) \Delta_{n-1,1},
$$

which with (27) gives

$$
w(1, n)=(-1)^{(n+1)(n+2) / 2} \cdot \frac{1}{k_{0}^{n+3}} .
$$

I shall now prove by mathematical induction that*

$$
w(m, n)=(-1)^{(n+1)(n+2) / 2+m-1} \cdot \frac{1}{k_{0}^{2 m+n+1}} .
$$

We see from (29) that the value of $w(m, n)$ given by (30) is correct for all values of $n$ if $m=1$.

I shall assume the correctness of formula (30) for all values of $n$ when $m=1,2,3, \cdots, p-1$, and prove, on that hypothesis, that it is correct for $m=p$, and hence for all $m$.

By a well known rule for multiplying a determinant by one of its minors,

$$
\Delta_{p-1, n+p}^{\prime}=\frac{\Delta_{p-2, n+p-1}^{\prime} \Delta_{p-2, n+p+1}^{\prime}-\left(\Delta_{p-2, n+p}^{\prime}\right)^{2}}{\Delta_{p-3, n+p}^{\prime}} .
$$

Then by (25) and what we have assumed, (31) may be written

$$
\Delta_{p-1, n+p}^{\prime}=\frac{(-1)^{(n+1)(n+2) / 2+p-1}\left[\frac{-\Delta_{n+p, p-1}}{\Delta_{n+p-1, p}}-\frac{\Delta_{n+p-1, p-1}^{2}}{\Delta_{n+p-1, p} \Delta_{n+p-2, p-1}}\right]}{-k_{0}^{2 p+n+2}\left(\Delta_{n+p-1, p-2} / \Delta_{n+p-1, p} \Delta_{n+p-2, p-1}\right)} .
$$

- Cf. Hadamard, Liouville's Journal, (4), vol. 8 (1892), §24, p. 138. Hadamard obtains (25) with $w(m, n)$ as in (30) but by a different method. 
But by the above mentioned rule for multiplying a determinant by one of its minors, the quantity within the bracket is equal to $-\Delta_{n+p-1, p-2} / \Delta_{n+p-2, p-1}$. Consequently, (32) may be written

$$
\Delta_{p-1, n+p}^{\prime}=\frac{(-1)^{(n+1)(n+2) / 2+p-1} \Delta_{n+p-1, p}}{k_{0}^{2 p+n+2}} .
$$

Comparison of (25) and (33) shows that the value of $w(m, n)$ as given by (30) is correct for $m=p$, as was to be proved.

As particular instances of (25) we have

$$
\begin{aligned}
\Delta_{m-1, m}^{\prime} & =(-1)^{m} \Delta_{m-1, m} / k_{0}{ }^{2 m}, \\
\Delta_{m-1, m-1}^{\prime} & =(-1)^{m-1} \Delta_{m-2, m} / k_{0}^{2 m-1} .
\end{aligned}
$$

Due to symmetry of the identity (21), the $\delta_{i}$ and the $k_{i}$ may be interchanged in (35), giving the relation

$$
\Delta_{m-2, m}^{\prime}=(-1)^{m-1} \Delta_{m-1, m-1} / k_{0}^{2 m-1} .
$$

We will now consider the continued fraction of the form (19) belonging to the normal* series

$$
\delta_{1}+\delta_{2} z+\delta_{3} z^{2}+\cdots
$$

If we denote by $a_{i}^{\prime}$ the $a_{i}$ obtained from (37), then by (34), (35), and (36) we obtain

$$
\begin{aligned}
a_{2 i}^{\prime} & =-\left(\Delta_{i-1, i}^{\prime}\right)^{2} /\left[\Delta_{i-1, i+1}^{\prime} \Delta_{i-2, i}^{\prime}\right]=\Delta_{i-1, i}^{2} /\left[\Delta_{i, i} \Delta_{i-1, i-1}\right]=a_{2 i+1}, \\
a_{2 i+1}^{\prime} & =\left(\Delta_{i-1, i+1}^{\prime}\right)^{2} /\left[\Delta_{i-1, i}^{\prime} \Delta_{i, i+1}^{\prime}\right]=-\Delta_{i, i}^{2} /\left[\Delta_{i-1,2} \Delta_{i, i+1}\right]=a_{2 i+2} .
\end{aligned}
$$

We have thus proved the following theorem:

THEOREM 1. If $k_{0}+k_{1} z+k_{2} z^{2}+\cdots$ is any normal power series giving rise to the continued fraction

$$
\frac{1}{a_{1}}+\frac{z}{a_{2}}+\frac{z}{a_{3}}+\cdots
$$

and $\delta_{0}+\delta_{1} z+\delta_{2} z^{2}+\cdots$ its reciprocal, then the continued fraction of the form (19) associated with the series $\delta_{1}+\delta_{2} z+\delta_{3} z^{2}+\cdots$ is

$$
\frac{1}{a_{2}}+\frac{z}{a_{3}}+\frac{z}{a_{4}}+\ldots \text {. }
$$

\footnotetext{
* A normal series is one giving rise to a normal Padé table.
} 
Chapter II. The convergence of the diagonal files for the series of Stieltjes

I pass now to the series of Stieltjes

$$
c_{0}-c_{1} z+c_{2} z^{2}-\cdots .
$$

The work of the preceding chapter is applicable here if we replace throughout $k_{2 i}$ by $c_{2 i}$ and $k_{2 i-1}$ by $-c_{2 i-1}$. In particular the continued fraction (16) becomes*

$$
G_{n}(z)+(-z)^{n}\left[\frac{1}{a_{1}{ }^{n}}+\frac{z}{a_{2}{ }^{n}}+\frac{z}{a_{3}{ }^{n}}+\cdots\right],
$$

where $G_{n}(z)=c_{0}-c_{1} z+\cdots+(-1)^{n-1} c_{n-1} z^{n-1}$ and the $a_{i}^{n}$ are obtained by replacing every $k_{i+n}$ by $(-1)^{i} c_{i+n}$ in (17). It is easily seen that now, in place of (17), we have

$$
\begin{aligned}
a_{2 i}^{n} & =\Delta_{i-1, i+n-1}^{2} /\left[\Delta_{i-1, i+n} \Delta_{i-2, i+n-1}\right], \\
a_{2 i+1}^{n} & =\Delta_{i-1, i+n}^{2} /\left[\Delta_{i-1, i+n-1} \Delta_{i, i+n}\right],
\end{aligned}
$$

in which it is to be understood that the $\Delta_{m, n}$ are the same as in Chapter I with every $k_{i}$ replaced by $c_{i}$.

In particular,

$$
\begin{aligned}
a_{2 i} & =A_{i}^{2} /\left(B_{i} B_{i-1}\right)=\Delta_{i-1, i-1}^{2} /\left(\Delta_{i-1, i} \Delta_{i-2, i-1}\right), \\
a_{2 i+1} & =B_{i}^{2} /\left(A_{i} A_{i+1}\right)=\Delta_{i-1, i}^{2} /\left(\Delta_{i-1, i-1} \Delta_{i, i}\right) .
\end{aligned}
$$

Thus each $a_{i}{ }^{n}$ is obtained from the corresponding $a_{i}$ by means of the substitution of $c_{i+n}$ for $c_{i}$ throughout.

Since, by hypothesis, $A_{n}$ and $B_{n}$, for $n=1,2,3, \cdots$, are positive, all the determinants $\Delta_{m, n}$ formed from them by removing the first $r$ rows and $r$ columns are positive. $\dagger$ Therefore, in (40), the bracketed part,

$$
\frac{1}{a_{1}^{n}}+\frac{z}{a_{2}^{n}}+\frac{z}{a_{3}^{n}}+\cdots
$$

is a continued fraction of Stieltjes with positive $a_{i}{ }^{n}$. We may therefore apply his convergence results for the continued fraction (2), bearing in mind that, as noted in the introduction, $z$ has been replaced by $1 / z$ in (2) and then the factor $z$ has been dropped. It is readily seen that if $P_{i}(z), Q_{i}(z)$ denote the numerator and denominator of the convergents of (2) (after $a_{i}$ has been re-

* Henceforth for convenience I write $a_{i}^{n}$ in place of $a_{i}^{(n)}$.

† Sylvester, Philosophical Magazine, (4), vol. 4 (1852), pp. 140-141. 
placed by $\left.a_{i}^{n}\right)$, and if $P_{i}^{\prime}(z), Q_{i}{ }^{\prime}(z)$ denote the corresponding numerators and denominators of (43), then*

$$
\begin{aligned}
P_{2 i}^{\prime}(z) & =z^{i-1} P_{2 i}\left(\frac{1}{z}\right), & Q_{2 i}^{\prime}(z) & =z^{i} Q_{2 i}\left(\frac{1}{z}\right), \\
P_{2 i+1}^{\prime}(z) & =z^{i} P_{2 i+1}\left(\frac{1}{z}\right), & Q_{2 i+1}^{\prime}(z) & =z^{i+1} Q_{2 i+1}\left(\frac{1}{z}\right) .
\end{aligned}
$$

The sequence of convergents accordingly separates into two sets, the even and the odd convergents. Stieltjes showed that when $\sum a_{i}^{n}$ is convergent, the numerators and denominators of the even convergents converge to limits $p(z), q(z)$ respectively; likewise those of the odd convergents to limits $p_{1}(z), q_{1}(z)$, and these limits are entire functions whose zeros all lie upon the negative half of the real axis. These functions are connected by the relation

$$
p_{1}(z) q(z)-p(z) q_{1}(z)=1 .
$$

On the other hand, when $\sum a_{i}^{n}$ is divergent, the even and the odd convergents converge to a common function $F(z)$ which is analytic over the entire plane excepting the whole or a part of the negative half of the real axis.

If now we denote the $i$ th convergent of $(40)$ by $U_{i}(z) / V_{i}(z)$, we have

$$
\frac{U_{i}(z)}{V_{i}(z)}=G_{n}(z)+(-z)^{n} \frac{P_{i}^{\prime}(z)}{Q_{i}^{\prime}(z)}=G_{n}(z)-(-z)^{n-1} \frac{P_{i}\left(\frac{1}{z}\right)}{Q_{i}\left(\frac{1}{z}\right)} .
$$

The odd and even convergents of (40) fill respectively the $n$th and $(n+1)$ st diagonal files of the Padé table. When $\sum a_{i}{ }^{n}$ is convergent, they have separate limits in consequence of the foregoing results of Stieltjes, and these limits are meromorphic over the entire plane except at $z=0$, with poles lying only upon the negative half of the real axis. If now we put

$$
\begin{aligned}
& u(z)=G_{n}(z) q\left(\frac{1}{z}\right)-(-z)^{n-1} p\left(\frac{1}{z}\right), \\
& v(z)=q\left(\frac{1}{z}\right),
\end{aligned}
$$

the limit of the even convergents $U_{2 i}(z) / V_{2 i}(z)$, for $i=\infty$, is $u(z) / v(z)$. Similarly the limit of the odd convergents will be $u_{1}(z) / v_{1}(z)$ where $u_{1}$ and $v_{1}$

* Cf. the formulas for $P_{i}(z), Q_{i}(z)$ given by Stieltjes, loc. cit., $\$ 2$. 
are the same as $u$ and $v$ except that $p, q$ are replaced by $p_{1}, q_{1}$. In consequence of (44) we have the relation

$$
u(z) v_{1}(z)-u_{1}(z) v(z)=(-z)^{n-1} .
$$

When $\sum a_{i}{ }^{n}$ is divergent, the application of the results of Stieltjes to (45) shows immediately that the two sets of convergents have a common limit which is analytic over the entire plane except the whole or a part of the negative real axis. We conclude therefore that whether $\sum a_{i}{ }^{n}$ converges or diverges every diagonal file of approximants to the right of the principal diagonal converges.

For a complete discussion of the relationship between the limits of these files we shall need to connect our successive series $\sum a_{i}^{n}$ with the primary series $\sum a_{i}$. For that purpose I shall now obtain expressions for the $a_{i}{ }^{n}$ in terms of the $a_{i}^{n-1}$.

Let the numerator and denominator of the $i$ th convergent of (40) be denoted by $U_{i}^{n}(z)$ and $V_{i}^{n}(z)$. Except for a common numerical factor, these may be obtained from (13) and (14). Thus, in particular, on setting $m=k$ and replacing $n$ by $n+k$ in (14) and introducing this factor, we have*

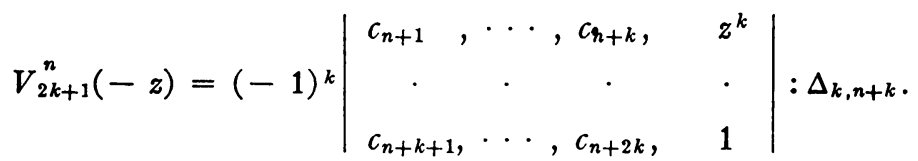

From (47) we see that the coefficient of $z^{k}$ in $V_{2 k+1}(z)$ is $\Delta_{k-1, n+k+1} / \Delta_{k, n+k}$. Now $V_{2 k+1}^{n}(z)$ is the denominator of the $(2 k+1)$ st convergent of the Stieltjes continued fraction (43), and it is easily proved by mathematical induction that the coefficient of $z^{k}$ is also $a_{1}^{n}+a_{3}^{n}+\cdots+a_{2 k+1}^{n}$. Therefore

$$
a_{1}^{n}+a_{3}^{n}+\cdots+a_{2 k+1}^{n}=\Delta_{k-1, n+k+1} / \Delta_{k, n+k} .
$$

If now we form by (41) the ratios $a_{2 i}^{n} / a_{2 i+1}^{n-1}$ and $a_{2 i+1}^{n} / a_{2 i+2}^{n-1}$ and then employ (48), we obtain the very fundamental relations $\dagger$

$$
a_{2 i}^{n}=\frac{a_{2 i+1}^{n-1}}{\sum_{i=0}^{i-1} a_{2 i+1}^{n-1} \sum_{i=0}^{i} a_{2 i+1}^{n-1}} \quad(n=1,2,3, \cdots)
$$

* Cf. Perron, loc. cit., p. 428. Note that Perron's $\Delta_{\mu \nu}$ differs by a factor $(-1)^{\mu(\mu+1) / 2}$ from that used by me.

$\dagger$ After this article was finished I found these relations (unused) in Stieltjes, loc. cit., $\$ 78$. 
and

$$
a_{2 i+1}^{n}=a_{2 i+2}^{n-1}\left(\sum_{i=0}^{i} a_{2 i+1}^{n-1}\right)^{2} .
$$

For $n=0$ we are to understand that $a_{i}^{0} \equiv a_{i}(i=1,2,3, \cdots)$.

Equation (49) may evidently be written in the form

$$
a_{2 i}^{n}=\frac{1}{\sum_{i=0}^{i-1} a_{2 i+1}^{n-1}}-\frac{1}{\sum_{i=0}^{i} a_{2 i+1}^{n-1}} .
$$

It now follows from (49) that when $\sum a_{2 i-1}^{n-1}$ is convergent and has the limit $L$, then from and after some index $i$,

$$
a_{2 i}^{n}<a_{2 i+1}^{n-1} /(L-e)^{2},
$$

where $e$ is an arbitrary fixed positive number. Therefore $\sum a_{2 i}^{n}$ is convergent when $\sum a_{2 i-1}^{n}$ is convergent. Furthermore, $\sum a_{2 i}^{n}$ is convergent even when $\sum a_{1-1}^{n}$ is divergent. For by summing (49a) for $i=1,2,3, \cdots, k$, we get

$$
\sum_{i=1}^{k} a_{2 i}^{n}=\frac{1}{a_{1}^{n-1}}-\frac{1}{\sum_{i=0}^{k} a_{2 i+1}^{n-1}}
$$

and this has the limit $1 / a_{1}^{n-1}$ when $k$ becomes infinite. We thus have proved the theorem:

Theorem 2. The successive series $\sum a_{2 i}^{n}, n=1,2,3, \cdots$, are all convergent.

We now turn to the series with odd subscripts, $\sum a_{2 i-1}^{n}$. We see at once by (50) that, when $\sum a_{i}^{n-1}$ is convergent,

$$
a_{2 i+1}^{n}<a_{2 i+2}^{n-1}\left(L^{\prime}\right)^{2} \quad(i=0,1,2, \cdots),
$$

where $L^{\prime}=\sum a_{2 i-1}^{n-1}$, and therefore $\sum a_{i}{ }^{n}$ is convergent. Taking $n=1,2,3, \cdots$ in succession, we obtain at once the following theorem:

THEOREM 3. If in the Stieltjes continued fraction (7) the series $\sum a_{i}$ is convergent, then all the associated series $\sum a_{i}{ }^{n}, n=1,2,3, \cdots$, are convergent.

Consider next the case in which $\sum a_{i}$ diverges. Then, while $\sum a_{2 i}^{1}$ will necessarily converge, $\sum a_{2 i-1}^{1}$ may either converge or diverge. On the former hypothesis all subsequent series $\sum a_{i}{ }^{n}, n=2,3,4, \cdots$, must converge, by application of Theorem 3. If $\sum a_{2 i-1}^{1}$ diverges, then as before $\sum a_{2 i}^{2}$ must converge but $\sum a_{2 i-1}^{2}$ may either converge or diverge, etc. Proceeding in this manner we perceive that in addition to the possibility of the convergence of 
all $\sum a_{i}^{n}, n=0,1,2, \cdots$, Case I, there are just two other possibilities: either, Case II, all of the series $\sum a_{i}{ }^{n}, n=0,1,2, \cdots$, will diverge; or else, Case III, the first $r$ will diverge while all subsequent to the $r$ th will converge.

It remains to see that both the last possibilities can be realized. For this purpose the following lemma will be established.

LEMMA 1. Let

$$
b_{1}+b_{2}+b_{3}+\cdots
$$

be any divergent series in which there is a limit to the ratio $b_{i} / b_{i-1}: \lim b_{i} / b_{i-1}=s$. Then if $s \geqq 1$ we have

$$
\lim _{i=\infty} \frac{b_{1}+b_{2}+\cdots+b_{i+k}}{b_{1}+b_{2}+\cdots+b_{i}}=s^{k} .
$$

According to the hypothesis, we may take a fixed $j$ sufficiently large to insure that $b_{j+k+i} / b_{j+i}(i \geqq 0)$ shall lie between the limits $s^{k}-e^{\prime} / 2$ and $s^{k}+e^{\prime} / 2$ where $e^{\prime}$ is an arbitrarily small prescribed positive quantity. Then

$$
\begin{aligned}
& \frac{b_{j+k}+b_{j+k+1}+\cdots+b_{j+k+\imath}}{b_{j}+b_{j+1}+\cdots+b_{j+i}} \\
& =\frac{1-\frac{b_{1}+b_{2}+\cdots+b_{j+k-1}}{b_{1}+b_{2}+\cdots+b_{j+k+i}}}{1-\frac{b_{1}+b_{2}+\cdots+b_{j-1}}{b_{1}+b_{2}+\cdots+b_{j+i}}} \cdot \frac{b_{1}+b_{2}+\cdots+b_{j+k+i}}{b_{1}+b_{2}+\cdots+b_{j+i}}
\end{aligned}
$$

will lie between the same limits inasmuch as the ratio of each term of the numerator of the left member to the corresponding term of the denominator lies between these limits. But since $\sum b_{i}=+\infty$, the first factor on the right by taking $i$ sufficiently large, say for $i>N$, becomes $1 /\left(1+e^{\prime \prime}\right)$, where $\left|e^{\prime \prime}\right|<e^{\prime}$. Thus

$$
\left|\frac{b_{1}+b_{2}+\cdots+b_{j+k+i}}{b_{1}+b_{2}+\cdots+b_{j+i}}-s^{k}\right| \leqq e^{\prime}\left(s^{k}+2\right)<\epsilon \quad \text { if } \quad e^{\prime}<\frac{\epsilon}{s^{k}+2}, i>N .
$$

The lemma accordingly results.

Construct now a Stieltjes continued fraction for which $\lim \left(a_{2 i+1} / a_{2 i-1}\right)=s$, $\lim \left(a_{2 i} / a_{2 i-2}\right)=r . \quad$ Let $0<r<1, s>1$. Then by the lemma and (49), (50) for $n=1$ we obtain

$$
\lim _{i=\infty}\left(a_{2 i+1}^{1} / a_{2 i-1}^{1}\right)=r s^{2}, \lim _{i=\infty}\left(a_{2 i}^{1} / a_{2 i-2}^{1}\right)=1 / s .
$$

If we so choose $s$ that $r s^{2}<1,1 / s<1$, then $\sum a_{i}$ will diverge while $\sum u_{i}{ }^{2}$ will converge: But if $r s^{2}>1,1 / s<1, \sum a_{i}^{1}$ as well as $\sum a_{i}$ will diverge. In 
the latter event, we may again apply the lemma and (49), (50) for $n=2$ and obtain

$$
\lim _{i=\infty}\left(a_{2 i+1}^{2} / a_{2 i-1}^{2}\right)=r^{2} s^{3}, \lim _{i=\infty}\left(a_{2 i}^{2} / a_{2 i-2}^{2}\right)=1 /\left(r s^{2}\right) .
$$

If we so choose $s$ that $r s^{2}>1, r^{2} s^{3}<1$, the series $\sum a_{i}, \sum a_{i}{ }^{1}$ will diverge, while $\sum a_{i}{ }^{2}$ will converge. But if $r^{2} s^{3}>1,1 /\left(r s^{2}\right)<1, \sum a_{i}, \sum a_{i}^{1}$, and $\sum a_{i}{ }^{2}$ will all diverge, and we may again apply the lemma, etc.

Continuing in this manner we find that if $0<r<1,1 / r^{(k-1) / k}<s<$ $1 / r^{k /(k+1)}$, all of the series $\sum a_{i}, \sum a_{i}^{1}, \cdots, \sum a_{i}^{k-1}$ will diverge, while the series $\sum a_{i}^{k}, \sum a_{i}^{k+1}, \cdots$, will converge. When $s \geqq 1 / r$, all of the series $\sum a_{i}^{n}$, $n=0,1,2,3, \cdots$, are divergent. Thus the two possibilities indicated above when $\sum a_{i}$ is divergent can be realized.

Whenever the Stieltjes series (5) is convergent, the series $c_{n}-c_{n+1} z$ $+c_{n+2} z^{2}-\cdots, n=1,2,3, \cdots$, are convergent, and therefore the numbers $1 /\left(a_{i}^{n} a_{i+1}\right), i=1,2,3, \cdots$, must be bounded for each $n=1,2,3, \cdots$.* Hence none of the associated series $\sum a_{i}^{n}, n=1,2,3, \cdots$, can be convergent.

These associated series may also diverge even when the Stieltjes series (5) is divergent. For consider the Stieltjes continued fraction for which $a_{2 i-1}$ $=1 /\left(i r^{i}\right), a_{2 i}=r^{i}, 0<r<1$. In this the numbers $1 /\left(a_{i} a_{i+1}\right)$ increase without $\operatorname{limit}$, and $\lim \left(a_{2 i+1} / a_{2 i-1}\right)=1 / r, \lim \left(a_{2 i+2} / a_{2 i}\right)=r$. Accordingly the series (5) and the associated series diverge.

We have seen that there are three cases which arise. Of these we will now consider the first.

Case I. The series $\sum a_{i}$ is convergent.

Then by Theorem 3 all the series $\sum a_{i}{ }^{n}, n=1,2,3, \cdots$, converge. Hence by the discussion at the beginning of the chapter all the diagonal files of approximants which lie to the right of the principal diagonal have limits which are meromorphic over the entire plane excepting $z=0$; with poles lying only on the negative half of the real axis; and the limits for no two successive files are identical.

To show that no two of these diagonal files have the same limit, I will show that for two of the denominators $v_{n}$ of these limits the roots of $v_{n}(1 / z)$ (different from 0 ) nearest the origin are distinct.

Stieltjes found that the roots of $Q_{2 k}(z)$ and $Q_{2 k+1}(z)$ for (2) are all real, non-positive, distinct, and separate each other. The former is of degree $k$ and has no root equal to zero, while the latter is of degree $k+1$ and has a zero root. The same is true of $Q_{2 k}^{n}(z)$, and $Q_{n k+1}^{n}(z)$, obtained from these by

* Cf. Stieltjes, loc. cit., §10. 
replacing $a_{i}$ by $a_{i}^{n}$. If then we denote by $r_{k}^{(n)}$ and $r_{k}^{(n+1)}$ the non-zero root nearest the origin of $Q_{2 k}^{n}(z)$ and $Q_{2 k+1}^{n}(z)$, we have

$$
r_{k}{ }^{(n)}>r_{k}{ }^{(n+1)} . \quad(n=1,2,3, \cdots ; k=1,2,3, \cdots) .
$$

But $r_{k}^{(n+1)}$ is also the non-zero root nearest the origin of $Q_{2 k}^{n+1}(z)$. If then we let $k \rightarrow \infty, r_{k}^{(n)}$ will approach a limit $R_{n} \neq 0$, and this limit is the non-zero root nearest the origin of the limit function $v_{n}(1 / z){ }^{*}$ We have

$$
R_{1} \geqq R_{2} \geqq R_{3} \geqq R_{4} \geqq \cdots .
$$

The equality sign can nowhere hold in (51), since, by (46), $v_{n}(1 / z)$ and $v_{n+1}(1 / z)$ cannot vanish for the same value of $z$ not zero, and therefore $R_{n} \neq R_{n+1}$ for all values of $n$. The distinctness of the functions $v_{n}(1 / z)$ therefore follows, and hence no two diagonal files can converge to the same limit.

Case II. All the series $\sum a_{i^{n}}, n=1,2,3, \cdots$, diverge.

Any two consecutive diagonal files to the right of and parallel to the principal diagonal converge to the same limit, and hence all these files have a common limit. The limit is analytic throughout the complex plane excepting the whole or a part of the negative half of the real axis.

Case III. The series $\sum a_{i}{ }^{n}, n=1,2,3, \cdots, r$, diverge while $\sum a_{i}^{r+j}$, $j=1,2,3,4, \cdots$, converge.

By the first part of the hypothesis the principal diagonal and all parallel files on its right up to the $(r+1)$ th diagonal file inclusive converge to one and the same limit, while by virtue of the second part of the hypothesis all the files beginning with the $(r+1)$ th converge to limits which are meromorphic over the entire plane except at $z=0$. Thus the limits of all these files are of the same meromorphic character, but the distinctness of the limits begins only on passing the $(r+1)$ th file.

We will now turn our attention to the diagonal files below the principal diagonal which start with an approximant on the vertical border of the Padé table. Instead of considering directly the convergence of these diagonal files of approximants, we may consider instead the convergence of their reciprocals which are files of approximants to the right of the principal diagonal of the Padé table for the reciprocal series of (5). Let this be

$$
d_{0}+d_{1} z+d_{2} z^{2}+\cdots .
$$

By (34) and (35) the determinants $A_{n}$ and $B_{n}$ with every $c_{i}$ replaced by $(-1)^{i} d_{i}$ are not all positive, but to the series

$$
d_{1}+d_{2} z+d_{3} z^{2}+\cdots
$$

* Stieltjes, loc. cit., \$19. 
belongs (Theorem 1, Chapter I) a Stieltjes continued fraction

$$
\frac{1}{a_{2}}+\frac{z}{a_{8}}+\frac{z}{a_{4}}+\ldots .
$$

Consequently (53) is a Stieltjes series and we may apply the results previously obtained for (7) and (43) using (53) as our primary series.

Three cases may be distinguished.

Case I. If the series $\sum a_{i}$ converges, the diagonal files of approximants for (53) to the right of the principal diagonal converge each to a different function, which is meromorphic except at $z=0$ with poles only on the negative half of the real axis. Consequently the diagonal files of approximants for (5) below the principal diagonal converge, each to a different function, which is meromorphic except at $z=0$ with zeros only on the negative half of the real axis.

If $\sum a_{i}$ is divergent, two cases may be distinguished corresponding to II and III above.

Case II. If all the diagonal files of approximants for (53) to the right of the principal diagonal have a common limit $F_{1}(z)$, then those below the principal diagonal for (5) have the limit $F(z)=1 /\left(d_{0}+z F_{1}\right)$, which is the limit of the continued fraction (7), and is analytic except over all or a part of the negative real axis.

Case III. If the first $r$ files of approximants of (53) to the right of the principal diagonal have the same limit, while after passing the $r$ th file the limits are distinct, then in the table of approximants of (5) the files below the principal diagonal will have the same limit until after passing the $(r+1)$ th file, whereupon they become distinct from one another. The limit functions are all meromorphic except at $z=0$ with zeros only on the negative half of the real axis.

One may ask the question whether, if the $r$ th and $(r+1)$ th diagonal files to the right of the principal diagonal have a common [different] limit [limits], a corresponding pair of adjacent files below the principal diagonal must have a common [different] limit [limits]. In Case I the answer is obviously in the affirmative, for then all the files to the right of and also those below the principal diagonal have distinct limits. In Case II when (5) converges, since its reciprocal also converges, both the series $\sum a_{n}^{n}, n=0$, $1,2,3, \cdots$, and the series similarly associated with (54) (which we shall denote for future reference by $\left.\sum \alpha_{i}^{n}, n=0,1,2, \cdots, \alpha_{i}^{0} \equiv a_{i+1}\right)$ must all diverge. Hence all the files have the same limit. Also for the divergent Stieltjes series for which $a_{2 i}=r^{i}, a_{2 i-1}=1 /\left(i r^{i}\right), 0<r<1$ (Chapter II) it is easily verified that $\sum a_{i}^{n}, \sum \alpha_{i}^{n}, n=0,1,2,3, \cdots$, all diverge. Finally, when 
$\lim \left(a_{2 i} / a_{2 i-2}\right)=r, \lim \left(a_{2 i+1} / a_{2 i-1}\right)=s, 0<r<1, s>1$, it may be easily seen that $a_{2 i+1} / a_{2 i-1}^{n}$ and $\alpha_{2 i+1}^{n+1} / \alpha_{2 i-1}^{n+1}$ both have the limit $r^{n} s^{n+1}$ for $i=\infty$. Consequently (except possibly when $\left.r^{n} s^{n+1}=1\right) \cdot \sum a_{i}^{n}$ and $\sum \alpha_{i}^{n+1}$ converge or diverge together. In all these examples pairs of adjacent files equally distant from the first diagonal file below the principal diagonal behave alike (i.e. the two files in each pair have a common limit or else they have distinct limits). In the following example pairs of adjacent files equidistant from the principal diagoñal behave alike. Choose $a_{2 i+3}=b_{i} /\left(a_{2}+a_{4}+\cdots+a_{2 i+2}\right)^{2}$, where $\sum b_{i}$ is any convergent positive-term series. Then $\sum \alpha_{2 i+1}^{1}=\sum b_{i}$ converges and hence by Theorem 3 all $\sum \alpha_{i}^{n}$ converge. But if $\sum a_{2 i}$ is divergent, $\sum a_{i}, \sum a_{i}^{1}$ are divergent, but $\sum a_{i}{ }^{2}$ and all subsequent series are convergent.

Now we shall examine the zeros and poles of the approximants $N_{m, n}(z) / D_{m, n}(z)$ of the Stieltjes series (5). If $n \geqq m$, these by (45) are equal to $G_{2 p+1}(z)-z^{2 p} p_{j}(1 / z) / Q_{j}(1 / z)$, where $j=2 m$ or $2 m+1$ according as $n-m=2 p$ or $2 p+1$; and if $n<m$ their reciprocals, by the discussion centering about (52), are equal to $d_{0}+d_{1} z+\cdots+d_{2 p+1} z^{2 p+1}+z^{2 p+1} P_{j}^{\prime \prime}(1 / z) / Q_{i}^{\prime \prime}(1 / z)$, where $P_{j}{ }^{\prime \prime} / Q_{i}{ }^{\prime \prime}$ is of the same form as $P_{j} / Q_{i}$ and $j=2 n$ or $2 n+1$ according as $m-n=2 p+1$ or $2 p+2$. If we replace $z$ by $1 / z$ and set $G_{n}^{1}(z)=d_{0}+d_{1} z$ $+\cdots+d_{n-1} z^{n-1}$, these become

$$
G_{2 p+1}\left(\frac{1}{z}\right)-\frac{1}{z^{2 p}} \frac{P_{j}(z)}{Q_{j}(z)} \quad \text { and } \quad G_{2 p}^{\prime}\left(\frac{1}{z}\right)+\frac{1}{z^{2 p+1}} \frac{P_{j}^{\prime \prime}(z)}{Q_{j}^{\prime \prime}(z)},
$$

where $P_{j}(z) / Q_{j}(z)$ and $P_{j}{ }^{\prime \prime}(z) / Q_{j}{ }^{\prime \prime}(z)$ are the $j$ th convergents of Stieltjes continued fractions of the form (2). Now it follows from the work of Stieltjes that when $n \geqq m-1$ the roots of the polynomials $D_{m, n}(z)$ are all distinct and lie on the negative half of the real axis. If $n \leqq m$ the same is true of the roots of $N_{m, n}(z)$. If $n=m$ or $m-1$, all the roots of $N_{m, n}(z)$ and $D_{m, n}(z)$ are real and alternate along the negative half of the real axis. Consider now the roots of the numerator and denominator of an arbitrary approximant $N_{m, n}(z) / D_{m, n}(z)$. In the above representation for it, $P_{j}(z) / Q_{j}(z)$ and $P_{i}{ }^{\prime \prime}(z) / Q_{i}{ }^{\prime \prime}(z)$ are monotone functions of $z$ which decrease from $+\infty$ to $-\infty$ as $z$ increases between two adjacent roots of the denominators.* But the polynomials $G_{n}(1 / z)$ and $G_{n}^{\prime}(1 / z)$ are continuous and bounded in any interval not containing the origin. Hence $G_{2 p+1}(1 / z)$ becomes equal to $P_{j}(z) / z^{2 p} Q_{j}(z)$ at least once between every two adjacent non-zero roots of $Q_{j}(z)$. Therefore if $n>m, N_{m, n}(z)$ vanishes at least once between every two adjacent roots of $D_{m, n}(z)$; similarly, if $n<m-1, D_{m, n}(z)$ vanishes at least once between every two adjacent roots of $N_{m, n}(z)$.

- Cf. Stieltjes, loc. cit., $\$ 3$. 


\section{Chapter III. Extended Stieltjes series}

I pass now to the question whether, given the Stieltjes series (5), it is possible to find a number $c_{-1}$ such that the series

$$
-c_{-1} z^{-1}+c_{0}-c_{1} z+c_{2} z^{2}-\cdots
$$

shall be a Stieltjes series, i.e. such that the determinants $A_{n}$ and $B_{n}$ in terms of the coefficients of (55) shall all be positive. The $B_{n}$ for (55), being the $A_{n}$ for the original series (5), are positive. Consequently it is both necessary and sufficient for the extension that a number $t=c_{-1}>0$ shall exist such that

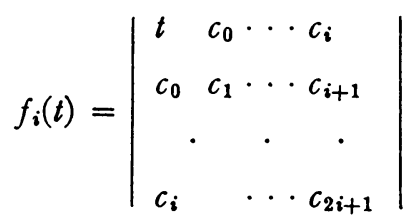

shall be positive for all values of $i=0,1,2,3, \ldots$.

Now

$$
f_{i}(t) \equiv \Delta_{i, i+1} \cdot t+f_{i}(0) .
$$

Since the coefficient of $t$ in (56) is positive, $c_{-1}$ must be taken greater than the root of $f_{i}(t)$, i.e. greater than

$$
t_{i}=-f_{i}(0) / \Delta_{i, i+1},
$$

for all values of $i$. It follows that the extension is possible when and only when the numbers $t_{i}, i=0,1,2, \cdots$, have an upper bound.

Now*

$$
t_{i}=a_{2}+a_{4}+\cdots+a_{2 i+2} .
$$

In fact, by the rule for multiplying a determinant by one of its minors

$$
f_{i}(t) \cdot \Delta_{i-1, i}=f_{i-1}(t) \Delta_{i, i+1}-\Delta_{i, i}^{2} .
$$

If we divide both members of this equation by $\Delta_{i-1, i} \Delta_{i, i+1}$ and then replace $i$ by $i-1, i-2, \cdots, 3,2,1,0$, we have with the aid of (42)

$$
\begin{aligned}
a_{2 i+2}= & f_{i-1}(t) / \Delta_{i-1, i}-f_{i}(t) / \Delta_{i, i+1}, \\
a_{2 i}= & f_{i-2}(t) / \Delta_{i-2, i-1}-f_{i-1}(t) / \Delta_{i-1, i}, \\
& \cdot \\
a_{4}= & f_{0}(t) / \Delta_{0,1}-f_{1}(t) / \Delta_{1,2} .
\end{aligned}
$$

* Stieltjes, loc. cit., $\$ 35$, eq. 11 , obtained this in a different manner. 
If to these equations we adjoin the identity

$$
a_{2}=t-f_{0}(t) / \Delta_{0,1},
$$

and then add them together and set $t=t_{i}$, we obtain (58).

It follows from (58) that the numbers $t_{i}, i=0,1,2, \cdots$, will have an upper bound when and only when the series $\sum a_{2 i}$ is convergent. When this condition is satisfied, we may take $c_{-1}=\sum a_{2 i}+\sigma$ where $\sigma$ is zero or an arbitrary positive number. We state this result in the following theorem.

Theorem 4. Given a Stieltjes series

$$
c_{0}-c_{1} z+c_{2} z^{2}-\cdots,
$$

there exists a Stieltjes series

$$
-c_{-1} z^{-1}+c_{0}-c_{1} z+c_{2} z^{2}-\cdots
$$

when and only when the series $\sum a_{2 i}$ in the Stieltjes continued fraction belonging to (5) is convergent.

When the condition is fulfilled the number $c_{-1}$ may be taken equal to $\sum a_{2 i}$ or any greater number.

Let us now suppose that $\sum a_{i}$ in (7) is convergent. Denote by*

$$
\frac{1}{a_{1}^{-1}}+\frac{z}{a_{2}^{-1}}+\frac{z}{a_{3}^{-1}}+\cdots
$$

the Stieltjes continued fraction belonging to (55), in which the $a_{n}^{-1}$ are necessarily positive. We must now express the $a_{n}^{-1}$ in terms of the $a_{n}$, which is obviously the same problem as that of expressing the $a_{i}{ }^{n-1}$ in terms of the $a_{i}{ }^{n}$. To do this we will need to develop some necessary formulas.

If we equate the two values of $t_{i-2}$ obtained from (57) and (58), and then advance the subscripts of every $c_{i}$ by $n$, we get the equation

$$
a_{2}{ }^{n}+a_{4}{ }^{n}+\cdots+a_{2 i-2}^{n}=-f_{i-2}^{n}(0) / \Delta_{i-2, n+i-1},
$$

where

Now

$$
f_{i-2}^{n}(0)=\left|\begin{array}{cccc}
0 & c_{n} & \cdots & c_{n+i-2} \\
c_{n} & c_{n+1} & \cdots & c_{n+i-1} \\
\cdot & & \cdot & \cdot \\
c_{n+i-2} & & \cdots & c_{n+2 i-3}
\end{array}\right| .
$$

$$
\Delta_{i-1, n+i-2}=f_{i-2}^{n}(0)+c_{n-1} \Delta_{i-2, n+i-1},
$$

\footnotetext{
* The superscripts as before are written for conven ence without parentheses.
} 
and therefore (61) may be written in the form

$$
\Delta_{i-1, n+i-2}=f_{i-2}^{n}(0) \cdot\left(c_{n-1}-\sum_{i=1}^{i-1} a_{2 i}^{n}\right) .
$$

By (41),

$$
\frac{a_{2 i}^{n-1}}{\Delta_{i-1, n+i-2}^{2}}=\frac{1}{\Delta_{i-1, i+n-1} \Delta_{i-2, i+n-2}} .
$$

Squaring the members of (63) and multiplying them into the corresponding members of (64), we have

$$
a_{2 i}^{n-1}=a_{2 i-1}^{n}\left(c_{n-1}-\sum_{i=1}^{i-1} a_{2 i}^{n}\right)^{2} \quad(i=2,3,4, \cdots) ;
$$

while for $i=1$ we have

$$
a_{2}^{n-1}=a_{1}^{n} c_{n-1}^{2}
$$

Also by (41), (63),

$$
a_{2 i+1}^{n-1}=\frac{a_{2 i}^{n}}{\left(c_{n-1}-\sum_{i=1}^{i-1} a_{2 i}^{n}\right)\left(c_{n-1}-\sum_{i=1}^{i} a_{2 i}^{n}\right)} \quad(i=2,3,4, \cdots),
$$

while for $i=0,1$ we have

$$
a_{1}^{n-1}=1 / c_{n-1} ; a_{3}^{n-1}=a_{2}^{n} /\left[c_{n-1}\left(c_{n-1}-a_{2}^{n}\right)\right] .
$$

Then we have, setting $n=0, c_{-1}=\sum a_{2 i}+\sigma, \sigma>0$, in (65), (67),

$$
a_{2 i}^{-1}=a_{2 i-1}\left(\sum a_{2 i}+\sigma-\sum_{1}^{i-1} a_{2 i}\right)^{2}<a_{2 i-1}\left(\sum a_{2 i}+\sigma\right)^{2},
$$

and

$$
a_{2 i+1}^{-1}=a_{2 i} /\left(\sum a_{2 i}+\sigma-\sum_{1}^{i-1} a_{2 i}\right)\left(\sum a_{2 i}+\sigma-\sum_{1}^{i} a_{2 i}\right)<a_{2 i} / \sigma^{2} .
$$

Hence since $\sum a_{i}$ is convergent, it follows that $\sum a_{i}^{-1}$ is also convergent.

We may now take $c_{-2}=\sum a_{2 i}^{-1}+\sigma^{\prime}$, where $\sigma^{\prime}>0$, and extend (55) to form the Stieltjes series

$$
c_{-2} z^{-2}-c_{-1} z^{-1}+c_{0}-c_{1} z+c_{2} z^{2}-\cdots,
$$

etc.

Continuing in this manner, we see that the series (5) can be extended indefinitely to the left. We have proved the following theorem: 
TheOREM 5. If in the Stieltjes continued fraction belonging to the Stieltjes series

$$
c_{0}-c_{1} z+c_{2} z^{2}-\cdots
$$

the series $\sum a_{i}$ converges, then the series (5) can be extended to the left by prefixing any finite or infinite number of terms: $-c_{-1} z^{-1}, \quad+c_{-2} z^{-2}, \quad-c_{-3} z^{-3}, \cdots$, in such wise that the extended series shall be Stieltjes series.

The coefficients in the prefixed terms are not unique.

Let us next suppose the series $\sum a_{i}$ to diverge. By Theorem $4, \sum a_{2 i}$ must converge in order that extension shall be possible, and consequently $\sum a_{2 i-1}$ is divergent. We then take $c_{-1}=\sum a_{2 i}+\sigma$, where $\sigma$ is zero or a positive number. I shall prove that unless $\sigma=0$, the series $\sum a_{2 i}^{-1}$ will be divergent, and therefore further extension will be impossible. In fact, by (65), if $\sigma \neq 0, a_{2 i}^{-1}>\sigma^{2} a_{2 i-1}, i=1,2,3, \cdots$, and therefore each term of $\sum a_{2 i} i^{-1}$ is greater than the corresponding term of the divergent series $\sigma^{2} \sum a_{2 i-1}$ so that extension is impossible.

When $\sigma \neq 0$, it has been shown that $\sum a_{2 i}{ }^{-1}$ is necessarily divergent. I shall give examples presently to show that when $\sigma=0, \sum a_{2 i} \bar{i}^{-1}$ may either converge or diverge. On the former hypothesis $\sum a_{2 i-1}^{-1}$ must be divergent, and therefore also $\sum a_{i}^{-1}$. For suppose the contrary. Then by Theorem 3, since $\sum a_{i}^{-1}$ converges, $\sum a_{i}, \sum a_{i}^{1}, \cdots$ converge. But by hypothesis $\sum a_{i}$ is divergent. We thus have a contradiction. Hence:

THEOREM 6. If in the Stieltjes continued fraction

$$
\frac{1}{a_{1}}+\frac{z}{a_{2}}+\frac{z}{a_{3}}+\cdots
$$

belonging to the Stieltjes series

$$
c_{0}-c_{1} z+c_{2} z^{2}-\cdots
$$

the series $\sum a_{i}$ diverges, and if (5) admits the extension

$$
\pm c_{-n} z^{-n} \mp c_{-n+1} z^{-n+1} \pm \cdots+c_{0}-c_{1} z+c_{2} z^{2}-\cdots,
$$

then the coefficients $c_{-1}, c_{-2}, \cdots, c_{-n}$ must be chosen successively in accordance with the relations

$$
\begin{aligned}
& c_{-i}=\sum_{2 k} a^{-i+1} \\
& c_{-n} \geqq \sum a_{2 k}^{-n+1} .
\end{aligned} \quad(i=1,2, \cdots, n-1),
$$

For examples illustrating the extension of Stieltjes series to the left the following lemma will be needed. 
LEMMA 2, Let $b_{1}+b_{2}+b_{3}+\cdots$ be any convergent series in which $\lim _{i=\infty}$ $b_{i} / b_{i-1}=r$; then if $0<r<1$,

$$
\lim _{i=\infty} \frac{b_{i+1}+b_{i+2}+\cdots}{b_{i+k+1}+b_{i+k+2}+\cdots}=\frac{1}{r^{k}} .
$$

According to the hypothesis we may take a fixed integer $m$ sufficiently large to insure that for all $n \geqq m$ the quotient $b_{n} / b_{n+k}$ will lie between $\left(1 / r^{k}\right)-\epsilon / 4$ and $\left(1 / r^{k}\right)+\epsilon / 4$, where $\epsilon$ is an arbitrarily small assigned positive number. Then

$$
\frac{b_{m}+b_{m+1}+\cdots+b_{m+p}}{b_{m+k}+b_{m+k+1}+\cdots+b_{m+k+p}}
$$

will lie between these same limits inasmuch as the terms are all of the same sign and the ratio of each term of the numerator to the corresponding term of the denominator lies between these limits. But since $\sum b_{i}$ is convergent there will be a limit to this fraction for $p \rightarrow \infty$ which lies between these limits.

As $\epsilon$ may be taken as small as desired by taking $m$ sufficiently large, the lemma accordingly results.

Construct now a Stieltjes continued fraction in which $a_{2 i}=r^{i}, \lim _{i-\infty}$ $a_{2 i+1} / a_{2 i-1}=s, 0<r<1, s>1$. As $\sum a_{i}$ diverges, $c_{-1}$ must be taken in accordance with (70). Then by (65) and (67), with $n=0$,

$$
\begin{aligned}
& \lim _{i=\infty} \frac{a_{2 i}^{-1}}{a_{2 i-2}^{-1}}=\lim _{i=\infty} \frac{a_{2 i-1}}{a_{2 i-3}} \frac{\left(a_{2 i}+a_{2 i+2}+\cdots\right)^{2}}{\left(a_{2 i-2}+a_{2 i}+\cdots\right)^{2}}=r^{2} s, \\
& \lim _{i=\infty} \frac{a_{2 i+1}^{-1}}{a_{2 i-1}^{-1}}=\lim _{i=\infty} \frac{a_{2 i}}{a_{2 i-2}} \frac{\left(a_{2 i-2}+a_{2 i}+\cdots\right)}{\left(a_{2 i+2}+a_{2 i+4}+\cdots\right)}=1 / r .
\end{aligned}
$$

If we now choose $r$ so that $r^{2} s<1$, then $\sum a_{2 i}{ }^{-1}$ will be convergent and both a first and second extension of (5) is possible. This requires that we have $r<1 / s^{1 / 2}<1$. Similarly, if $r^{2} s<1$, we have

$$
\begin{aligned}
& \lim _{i=\infty} \frac{a_{2 i}^{-2}}{a_{2 i-2}^{-2}}=\lim _{i=\infty} \frac{a_{2 i-1}^{-1}}{a_{2 i-3}^{-1}} \frac{\left(a_{2 i}^{-1}+\cdots\right)^{2}}{\left(a_{2 i-2}^{-1}+\cdots\right)^{2}}=r^{3} s^{2}, \\
& \lim _{i=\infty} \frac{a_{2 i+1}^{-2}}{a_{2 i-1}^{-2}}=\lim _{i=\infty} \frac{a_{2 i}^{-1}}{a_{2 i-2}^{-1}} \frac{\left(a_{2 i-2}^{-1}+\cdots\right)}{\left(a_{2 i+2}^{-1}+\cdots\right)}=1 /\left(r^{2} s\right) .
\end{aligned}
$$

Then if $r^{3} s^{2}<1$, that is, if $r<1 / s^{2 / 3}$, a third extension is possible and $\lim a_{2 i} \bar{i}^{3} / a_{2 i-2}^{-3}=r^{4} s^{2}$, etc. Thus, if 


$$
\frac{1}{s^{k /(k+1)}}<r<\frac{1}{s^{(k-1) / k}},
$$

the series $\sum a_{2 i}, \sum a_{2 i^{-1}}, \cdots, \sum a_{2 i^{-k+1}}$ will converge, while $\sum a_{2 i^{-k}}$ is divergent. Hence extension of (5) to the left for $k$ terms is then possible, but a further extension is impossible. If $r=1 / s$, an extension of infinite order is possible.

The following theorem follows immediately from (49), (50) of the preceding chapter.*

THEOREM 7. Given any series of positive numbers $\sum a_{i}$, there is uniquely determined a power series of Stieltjes

$$
c_{0}-c_{1} z+c_{2} z^{2}-\cdots
$$

\section{belonging to the Stieltjes continued fraction}

$$
\frac{1}{a_{1}}+\frac{z}{a_{2}}+\frac{z}{a_{3}}+\cdots
$$

The $c_{i}$ are given in terms of the $a_{i}$ by the following formulas:

$$
\begin{aligned}
c_{i} & =1 / a_{1}^{i} \\
a_{2 k}^{i} & =a_{2 k+1}^{i-1} / \sum_{k=1}^{k} a_{2 k-1}^{i-1} \sum_{k=1}^{k+1} a_{2 k-1}^{i-1}, \\
a_{2 k-1}^{i} & =a_{2 k}^{i-1}\left(\sum_{k=1}^{k} a_{2 k-1}^{i-1}\right)^{2} .
\end{aligned} \quad(i=0,1,2, \cdots),
$$

Chapter IV. A particular example

The continued fraction $\dagger$

$$
\frac{1}{1}+\frac{z}{1}+\frac{z}{1}+\frac{z}{1}+\cdots .
$$

I will determine the $c_{i}$ and the $a_{i}{ }^{n}$ (which are the same as the $\alpha_{i}^{n}$ in this case) for the above continued fraction. I will show that the radius of convergence of the corresponding Stieltjes series is $1 / 4$. The associated series $\sum a_{i}^{n}, n=1,2,3, \cdots$, are divergent, but the $\sum a_{2 i}^{n}$ converge, and consequently

$$
c_{n}=\sum a_{2 i}^{n+1} \quad(n=0,1,2,3, \cdots)
$$

* Stieltjes, Annales de Toulouse, vol. 3, H, pp. 1-17, 1889, shows the one-to-one correspondence between his series and continued fractions. He obtains the $c_{i}$ in terms of the $a_{i}$ by a different method.

† Laurent, Note sur les fractions continues, Nouvelles Annales de Mathématiques, (2), vol. 5 (1866), pp. 540-552. 
The $c_{n}$ being known, (72) serve to give the sums of certain positive-term series.

To determine the $c_{i}$, write

Hence,

$$
F_{1}(z)=1+\frac{z}{1}+\frac{z}{1}+\cdots=1+\frac{z}{F_{1}(z)} .
$$

and

$$
F_{1}(z)=\frac{1+(1+4 z)^{1 / 2}}{2}
$$

$$
\begin{aligned}
F(z) & =\frac{1}{1}+\frac{z}{1}+\frac{z}{1}+\cdots \\
& =\left(F_{1}-1\right) / z \\
& =\frac{-1+(1+4 z)^{1 / 2}}{2 z} .
\end{aligned}
$$

If we develop the numerator of the last expression in a Taylor's power series about $z=0$, we may then divide by $2 z$ and obtain

$$
F(z)=c_{0}-c_{1} z+c_{2} z^{2}-\cdots,
$$

where

$$
\begin{aligned}
c_{2 k} & =\frac{(2 k+3)(2 k+5) \cdots(4 k-1) 2^{k}}{k !}, \\
c_{2 k+1} & =\frac{(2 k+3)(2 k+5) \cdots(4 k+1) 2^{k}}{k+1 !},
\end{aligned}
$$

and therefore satisfy the recurrence relations

$$
\begin{aligned}
c_{2 k+1} & =[(4 k+1) /(k+1)] c_{2 k}, \\
c_{2 k} & =2[(4 k-1) /(2 k+1)] c_{2 k-1}, \\
c_{0} & =1 .
\end{aligned}
$$

Since $\lim \left(c_{i} / c_{i-1}\right)=4$ it follows that the radius of convergence of (73) is equal to $1 / 4$.

We will next get the elements in the associated continued fractions. Using (49), (50):

$$
\begin{aligned}
a_{2 k}^{1} & =1 /[k(k+1)], \\
a_{2 k+1}^{1} & =(k+1)^{2} .
\end{aligned}
$$


Then since $1^{2}+2^{2}+\cdots+(k+1)^{2}=(k+1)(k+2)(2 k+3) / 2 \cdot 3$, we have

$$
\begin{aligned}
a_{2 k}^{2} & =(2 \cdot 3)^{2} /[k(k+2)(2 k+1)(2 k+3)], \\
a_{2 k+1}^{2} & =(k+1)(k+2)(2 k+3)^{2} /(2 \cdot 3)^{2} .
\end{aligned}
$$

The next step will involve the formula $1 \cdot 2 \cdot 3^{2}+2 \cdot 3 \cdot 5^{2}+\cdots+(k+1)$ $(k+2)(2 k+3)^{2} \equiv(k+1)(k+2)(k+3)(2 k+3)(2 k+5) / 5$. We have

$$
\begin{aligned}
\stackrel{8}{a_{2 k}} & =(2 \cdot 3)^{2}(5)^{2} /[k(k+1)(k+2)(k+3)(2 k+1)(2 k+5)], \\
a_{2 k+1}^{3} & =(k+1)(k+2)^{2}(k+3)(2 k+3)(2 k+5) /\left[(2 \cdot 3)^{2}(5)^{2}\right] .
\end{aligned}
$$

In general:

$$
\begin{aligned}
a_{2 k+1}^{2 i+1} & =\frac{(k+1) \cdots(k+i)(k+i+1)^{2}(k+i+2) \cdots(k+2 i+1)(2 k+3) \cdots(2 k+4 i+1)}{(2 \cdot 3)^{2}(5)^{2}(2 \cdot 7)^{2} \cdots[2(4 i-1)]^{2}(4 i+1)^{2}}, \\
a_{2 k}^{2 i+1} & =\frac{(2 \cdot 3)^{2}(5)^{2}(2 \cdot 7)^{2} \cdots[2(4 i-1)]^{2}(4 i+1)^{2}}{k \cdots(k+2 i+1)(2 k+1) \cdots(2 k+2 i-1)(2 k+2 i+3) \cdots(2 k+4 i+1)}, \\
a_{2 k+1}^{2 i} & =\frac{(k+1) \cdots(k+2 i)(2 k+3) \cdots(2 k+2 i-1)(2 k+2 i+1)^{2}(2 k+2 i+3) \cdots(2 k+4 i-1)}{(2 \cdot 3)^{2}(5)^{2}(2 \cdot 7)^{2} \cdots(4 i-3)^{2}[2(4 i-1)]^{2}}, \\
a_{2 k}^{2 i} & =\frac{(2 \cdot 3)^{2}(5)^{2}(2 \cdot 7)^{2} \cdots(4 i-3)^{2}[2(4 i-1)]^{2}}{k \cdots(k+i-1)(k+i+1) \cdots(k+2 i)(2 k+1) \cdots(2 k+4 i-1)} .
\end{aligned}
$$

These formulas are seen to hold for all $k$ when $i=1$. Assuming them true for all $k$ and for all $i \leqq I-1$, they may, with the aid of the following formulas, be proved* for $i=I$. Set

$$
\begin{aligned}
Y_{k}^{i}=(k+1) & \cdots(k+i-1)(k+i)^{2}(k+i+1) \cdots(k+2 i-1)(2 k+3) \\
& \cdots(2 k+4 i-3), \\
Z_{k}^{i}=(k+1) & \cdots(k+2 i)(2 k+3) \cdots(2 k+2 i-1)(2 k+2 i+1)^{2}(2 k+2 i+3) \\
& \cdots(4 k+4 i-1) .
\end{aligned}
$$

Then,

$$
\begin{gathered}
\sum_{k=0}^{k} Y_{k}^{i}=(k+1) \cdots(k+2 i)(2 k+3) \cdots(2 k+2 i-1)(2 k+2 i+1)^{2}(2 k+2 i+3) \\
\cdots(2 k+4 i-1) /(2(4 i-1)), \\
\sum_{k=0}^{k} Z_{k}^{i}=(k+1) \cdots(k+2 i+1)(2 k+3) \cdots(2 k+4 i+1) /(4 i+1) .
\end{gathered}
$$

* The completion of the proof, which offers no difficulty, will be omitted.

UNTVERSITY OF WISCONSIN,

Madison, Wis. 